\title{
Tres holotipos "olvidados" de la Colección de Aves del Museo Nacional de Ciencias Naturales de Madrid
}

\author{
F. Hernández Carrasquilla (*)
}

\section{Introducción}

Durante un trabajo de revisión de la colección de pieles de aves del Museo Nacional de Ciencias Naturales de Madrid se hallaron los holotipos de tres subespecies cuya presencia había pasado desapercibida previamente. Ya que el Código Internacional de Nomenclatura Zoológica (Rec. $72 \mathrm{G} 4$ ) recomienda la divulgación del material típico que posee cada institución (ICZN, 1985), hemos decidido publicar la información más relevante que poseemos de estos ejemplares, sin entrar en consideraciones sobre su validez taxonómica, basándonos en el trabajo original del autor.

La información de cada ejemplar-tipo se presenta de la manera formal recomendada actualmente en estudios taxonómicos (Mayr \& Ashlock, 1991). Los autores cuando propusieron estas nuevas subespecies no hicieron una descripción propiamente dicha de la misma sino tan sólo un diagnóstico, por ello no aparece recogido ese apartado. Los tres tipos se encuentran depositados en el Museo Nacional de Ciencias Naturales de Madrid.

\section{SigLAS UTILIZADAS:}

MNCN: Museo Nacional de Ciencias Naturales de Madrid

$\sigma^{2}$ : Macho

Ad: Adulto

\section{Descripción de los tipos}

\author{
Clase AVES \\ Orden CHARADRIIFORMES \\ Familia ALCIDAE
}

Genus Uria Brisson, 1760

Uria aalge (Pontoppidan, 1763)

Uria aalge ibericus Bernis, 1948

Holotipo: piel de estudio, MNCN 5125. $0^{x} \mathrm{Ad}$. Colectado el 16 de Junio de 1948 por Francisco Bernis Madrazo.

LOCALIDAD TíPICA: Furna del Castelete, Sisarga Mayor, Islas Sigargas, La Coruña, Comunidad Gallega, España.

DisTRIBUCIÓN: Península Ibérica.

ETIMOLOGÍA: el nombre hace referencia a su área de distribución.

Medidas Del holotipo (mm): longitud del ala: 187, altura del pico: 13, longitud del pico: 46.

PARATIPOS: no hay ninguno definido, si bien el autor hace mención de varios ejemplares (12 en concreto) del MNCN (algunos hoy perdidos) que pertenecerían a esta subespecie y podrían considerarse como tales (serían las pieles de estudio MNCN 5127, 5128, 5129).

MATERIAL REVISADO POR EL AUTOR: 15 ejemplares de la especie depositados en el MNCN

DiAGNOSIS: “...plumaje de color muy próximo, pero menos grisáceo que la raza típica, difiriendo en ese particular por lo menos tanto como de ésta, de la oscura raza albionis. Y de ambas razas citadas difiere por sus medidas menores".

Comentario: El Arao Común presenta una compleja variación geográfica que afecta tanto a la talla (Jones, 1988) como a diversas características del plumaje (oscuridad del manto, porcentaje de individuos "bridados" en las colonias, etc.) (Cramp, 1985). Esta variación clinal se ha simplificado en varias subespecies cuya validez está en perpetua discusión. Según qué autor se consulte, el número de subespecies oscila entre 2 (Wijs, 1978) y 7 (ver

\footnotetext{
* Colección de Aves y Mamíferos. Museo Nacional de Ciencias Naturales. C/ José Gutiérrez Abascal, 2 E-28006 Madrid
} 
Vaurie, 1965). La taxonomía más aceptada considera sólo 3 subespecies: $U$. a. hyperborea Salomonsen, 1932, U. a. aalge (Pontoppidan, 1763) englobando a $U$. a. intermedia (Nilsson, $1835)$ y a $U$. a. spiloptera Salomonsen, 1932 y $U$. a. albionis Witherby, 1923 que incluye a U. a. ibericus (Glutz von Blotzheim \& Bauer, 1982). No existen trabajos recientes sobre la validez de esta subespecie.

\section{Clase AVES \\ Orden PASSERIFORMES \\ Familia ALAUDIDAE \\ Genus Galerida Boie, 1828 \\ Galerida theklae Brehm , 1858}

Galerida theklae berengueri Cabrera, 1922

Holotipo: piel de estudio. MNCN 11298. $0^{x}$ Ad. Colectado por Angel Cabrera el 13 de Noviembre de 1921. Fue preparado por José Luis Bernaldo de Quirós.

LOCALIDAD TíPICA: Yebala, llano de Uad Martín, cerca de Dar-es-Skiek (proximidades de la actual Chefchaouene) al sur de Tetuán, Marruecos.

DisTRIBUCIÓN: noreste de Marruecos.

Etimología: dedicada al Alto Comisario de España en Marruecos (suponemos que su apellido sería Berenger).

MedidAs DEL HOLOTIPO (mm): longitud total: 170, longitud del ala: 108, longitud del tarso: 28, longitud del culmen: 13 .

TOPOTIPO: piel de estudio. MNCN 11309. $0^{\pi}$. Colectado en la misma localidad el día 9 de Noviembre de 1921.

MATERIAL REVISADO POR EL AUTOR: topotipos de erlangueri Hartert, 1904, harterti Erlanger, 1894 y ruficolor Withaker, 1898.

DiAgNosis: "Timoneras centrales obscuras, parecida a erlangueri en su plumaje obscuro, pero un poco más robusta y con un ancho borde ante rosáceo bien definido en las remeras secundarias, que la hacen parecer a primera vista más clara de lo que realmente es. El color general, menos amarillento que en la G. t. theklae, sobre todo en las partes inferiores. Manchas pectorales grandes, negras".

COMENTARIO: Galerida theklae presenta una variación clinal muy marcada en su rango de distribución. La taxonomía de las poblaciones norteafricanas esta bastante oscurecida por el gran número de subespecies descritas en ese área (12). En Marruecos, Vaurie (1959) acepta erlangueri en el norte (aguirrei y berengueri serían consideradas sinonimias), ruficolor en el sur (con harterti y theresae Meinertzhagen, 1939 como sinonimias) y superflua Hartert, 1897 en el este (con cyrenaica Withaker, 1902, hilgerti Rothschild \& Hartert, 1912, Alauda cristata pallida Withaker, 1895 y Galerida cristata carolinae Erlanger, 1897 como sinonimias). Howard \& Moore (1990) presentan un criterio semejante a este. Abs (1963) en un trabajo sobre la evolución del género Galerida no menciona estas dos subespecies. Roseelar en Cramp (1988) no considera válida la subespecie $G$. $t$. berengueri, al igual que Keith et al. (1992).

\section{Clase AVES \\ Orden PASSERIFORMES \\ Familia ALAUDIDAE \\ Genus Galerida Boie, 1828 \\ Galerida theklae Brehm , 1858 \\ Galerida theklae aguirrei Cabrera, 1922}

Holotipo: piel de estudio. MNCN 11303. $\sigma^{\pi}$ Ad. Colectado por Angel Cabrera el 10 de Mayo de 1919.

LOCALIDAD TÍPICA: Llanura de Garet, al este del Monte Arruí, Rif Oriental, Marruecos.

DisTRIBUCIÓN: Rif Oriental, Marruecos.

Etimología: en honor del Jefe de la sección de Marruecos, D. Manuel Aguirre de Cárcer.

MEdidAS DEL HOLOTIPO (mm): longitud del ala: 102, longitud de la cola: 54, longitud del tarso: 25.10, longitud del pico: 15.62 .

PARATIPO: piel de estudio. MNCN 11305. $0^{\top}$ Ad. Colectado en la misma localidad y fecha que el holotipo por Angel Cabrera.

MATERIAL REVISADO POR EL AUTOR: topotipos de erlangueri, harterti y ruficolor.

DiAGNOSIS: “...plumaje más pálido que erlangueri o berengueri, rectrices centrales rojizas, casi como la porción rojiza de las externas. En este carácter coincidiría con ruficolor pero se separaría de ella por carecer de un borde pálido en las secundarias y por las manchas pectorales estrechas".

COMENTARIO: En general podemos remitirnos a los comentarios vertidos en la subespecie anterior. Roseelar en Cramp (1988), sin embargo, considera a $G$. $t$. aguirrei como subespecie válida aunque confunde su área de distribución con la de ruficolor. Igual error comenten Keith et al. (1992) en su trabajo sobre las aves de África.

El autor incluye una clave para separar las 4 formas marroquíes de G. theklae: 
a. Plumaje obscuro, timoneras centrales mucho más obscuras que la parte rojiza de las timoneras externas.

b. remeras secundarias sin un ancho borde pálido, abdomen ante claro .............................erlangueri

b'. remeras secundarias con un ancho borde pálido, abdomen ante rosáceo ........................... berenguer

a'. Plumaje más pálido, timoneras centrales rojizas, caso como la porción rojiza de las externas.

c. un ancho borde pálido en las secundarias, manchas pectorales anchas ................................ ruficolor

c'. sin un ancho borde pálido en las secundarias, manchas pectorales estrechas aguirrei

\section{AGRADECIMIENTOS}

Agradezco a D ${ }^{\text {a }}$ Josefina Barrerio (Conservadora de Aves y Mamíferos del MNCN) y a la Dra. Eulalia Moreno (Vicedirectora de Colecciones del $\mathrm{MNCN}$ ) su apoyo entusiasta. El manuscrito se ha visto mejorado gracias a los comentarios de un revisor anónimo.

\section{Referencias}

ABS, M., 1963. Vergleichende Untersuchungen an Haubenlerche (Galerida cristata) und Theklalerche (Galerida theklae). Bonn. Zoologische Beiträge, 14: $1-128$

BERNIS, F., 1948. Las aves de las islas Sisargas en junio. Bol. R. Soc. Esp. Hist. Nat., 49: 647-684.

CABrera, A., 1922. Una excursión de dos meses por Yebala. Bol. R. Soc. Esp. Hist. Nat., 22: 101-113.

Cramp, S. (Ed.), 1985. The Birds of the Western Palearctic. Vol. 4. Oxford University Press. Oxford. 960 pp.

Cramp, S. (Ed.), 1988. The Birds of the Western Palearctic. Vol. 5. Oxford University Press. Oxford. $1063 \mathrm{pp}$.

Glutz von Blotzheim, U. N. \& Bauer, K., 1982. Handbuch der Vögel Mittleleuropas. Akademische Verlagsgesellschaft. Wiesbaden. 1270 pp.
Howard, R. \& MoOre, A., 1990. A complete checklist of the birds of the World. Academic Press. London. 630 pp.

INTERNATIONAL COMMISSION ON ZOOLOGICAL NomenClatURE (ICZN), 1985. International Code of Zoological Nomenclature. International Trust of Zoological Nomenclature. University of California Press. Berkeley. 388 pp.

JONES, P. H., 1988. The european cline in wing-length of Guillemots Uria aalge. Seabird, 11: 19-21.

Keith, G. S., Urban, E. K. \& Fry, C. H., 1992. The Birds of Africa. Volumen IV. Academic Press, London. 609 pp.

Mayr, E. \& Ashlock, P. D., 1991. Principles of systematic zoology. McGraw-Hill Inc. New York. 475 pp.

VAurie, C., 1959. The birds of the Palearctic fauna. Passeriformes. H. F. \& G. Witherby LTD. London. $762 \mathrm{pp}$.

VAurie, C., 1965. The birds of the Palearctic fauna. No Passeriformes. H. F. \& G. Witherby LTD. London. $763 \mathrm{pp}$.

WiJs, W. J. R., 1978. Die geografiesk variatie van de Zeekoet (Uria aalge, Pontoppidan) en de mogelijke relatie hiervan met de Laat-Pleistocene geschiedenis van de noordelijke Oceaan. Tesis Doctoral, Universidad de Amsterdam. 160 pp.
Enviado, el 16-VII-1998 Aceptado, el 16-XI-1998 Publicado, el 31-XII-1998 